\title{
Why titanium alloys withstand more strain under dwell-fatigue than under fatigue?
}

\author{
S. Hémery ${ }^{1}$, A. Naï-Ali ${ }^{1}$, C. Lavogiez ${ }^{1}$, P. Villechaise ${ }^{1}$ \\ ${ }^{1}$ Institut Pprime, CNRS - ISAE-ENSMA - Université de Poitiers, UPR CNRS 3346, Physics and Mechanics of Materials Department, ENSMA - Téléport 2, 1 avenue Clément Ader, BP \\ 40109, 86961 Futuroscope Chasseneuil Cedex, France \\ * Corresponding author: samuel.hemery@ensma.fr
}

\begin{abstract}
$\underline{\text { Abstract }}$
Experimental observations reveal that crack initiation proceeds at a reduced number of cycles if a load hold at maximum stress is introduced during cyclic testing. Although this feature was extensively investigated and stems from the occurrence of room temperature creep, other differences between fatigue and dwell-fatigue behaviors are still to be clarified. In particular, a higher plastic strain is cumulated prior to failure if the load hold is present. This observation highlights differences in the deformation behavior depending on the applied loading. The present article reports an investigation of this point using Fast-Fourier transforms based crystal plasticity simulations of the stress and strain field. Significant differences were evidenced and discussed in light of experimental results reported in the literature. In particular, the occurrence of crack initiation for different cumulated plastic strain depending on the loading conditions is elucidated.
\end{abstract}

\section{Introduction}

Titanium alloys are widely used in the aerospace industry owing to superior properties such as a high specific strength or a good corrosion resistance. The fatigue performance is also a critical concern for numerous applications. In titanium alloys, the fatigue crack initiation stage is known to account for roughly $90 \%$ of the total fatigue life [1]. Hence, the understanding of the processes involved in the crack formation has been of continuous interest in order to improve both the predictability of fatigue failure and microstructures to enhance understanding of the processes involved in the crack formation has been of continuous interest in order to improve both the predictability of fatigue failure and microstructures to enhance reduction in the number of cycles to failure [2]. Room temperature creep, also called cold creep, has been evidenced as a key process for the early crack initiation. Numerous experimental reduction in the number of cycles to failure [2]. Room temperature creep, also called cold creep, has been evidenced as a key process for the eaty
and simulation investigations enabled to identify the underlying mechanisms and the microstructural features involved in crack initiation [3-5].

Studies focusing on fatigue crack initiation evidenced a critical role of basal slip [6,7]. In contrast, a hard - soft grain pair, where load shedding from soft grains to hard grains and stress concentration induced by dislocation pile ups are expected, was reported to play a key role and favor quasi-cleavage facet formation in hard grains [2]. This difference in mechanisms is also accompanied by a transition from surface to sub-surface crack initiation with the introduction of the load hold.

Several features of the so-called dwell effect still remain poorly understood. In particular, the cumulated plastic strain magnitude sustained under fatigue loading for a given alloy is significantly lower than the cumulated plastic strain sustained under dwell-fatigue loading [8]. An example of cumulated plastic strain - number of cycles plot shown on figure 1 for Tiis significantly lower than the cumulated plastic strain sustained under

These considerations implies that, in spite of a similar cumulated plastic strain, conditions for fatigue crack initiation are not met under a dwell-fatigue loading. This was experimentally confirmed using fatigue and dwell fatigue tests interrupted after $1 \%$ plastic strain (not shown in the present article). Although initiated cracks are noticed on the surface of the fatigue tested specimen, no such cracks are noticed on the dwell-fatigue tested specimen. This reveals significant differences in the deformation behavior of titanium alloys submitted to fatigue and dwell-fatigue loadings.

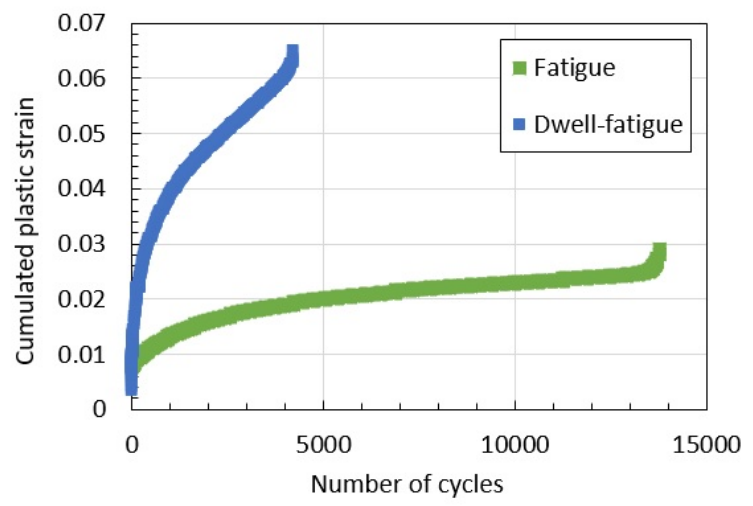

Figure 1. Cumulated plastic strain plotted with respect to the number of cycles for fatigue and dwell-fatigue loadings

The present article reports an investigation of this difference in deformation behavior. A Fast-Fourier transforms-based crystal plasticity model was used to simulate the stress and strain fields in a Ti-6Al-4V microstructural patch. This technique was used because it combines an easy generation of aggregates from EBSD data and an efficient calculation of yet computationally expensive simulations of fatigue and dwell-fatigue tests. Differences in terms of stress and strain fields are analyzed at a similar total strain and discussed regarding the
crack initiation behavior.

\section{Materials and simulations}

Ti-6Al-4V with a bi-modal microstructure was used as a reference material. The duplex microstructure is composed of primary alpha nodules, which account for $36 \%$ of the surface fraction, and secondary alpha lamellas embedded in a beta matrix. The average nodule size is about $13 \mu \mathrm{m}$. An aggregate was generated based on a region characterized using electron back-scattered diffraction.

The region of interest is roughly $400 \times 400 \mu \mathrm{m}^{2}$. It was characterized using a JEOL 6100 SEM equipped with an EBSD detector provided by EDAX. A squared step of $0.4 \mu \mathrm{m}$ was used to accurately describe both nodules and lamellar colonies. The local crystallographic orientation relative to the loading direction of the simulated experiments is shown on figure 2 a with an inverse pole figure map superimposed with the image quality. This region is weakly textured according to the orientation density function plotted on an inverse pole figure shown in figure $2 \mathrm{~b}$. The $2 \mathrm{D}$ map was then extruded over 10 layers to create a quasi-3D aggregate. In order to statistically simulate the effect of the microstructure underneath the surface, 10 layers, where each voxel is assigned a random crystallographic orientation taken from the surface layer, were merged with it. 

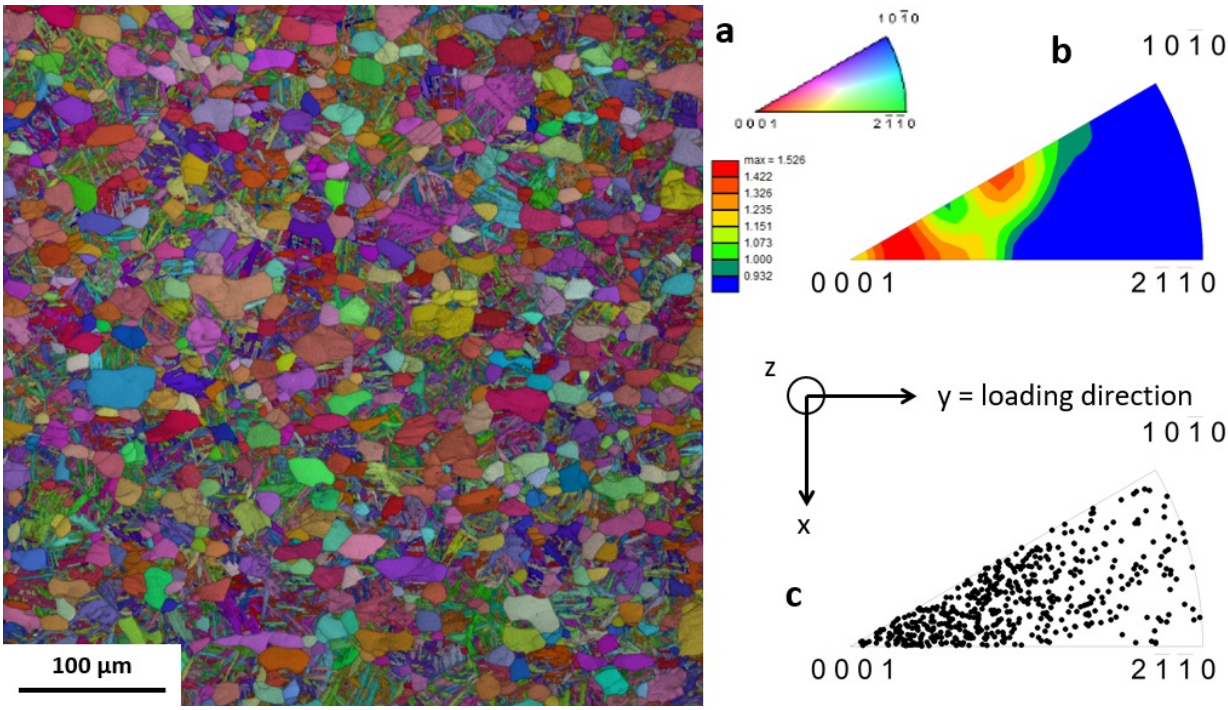

Figure 2 a. Inverse pole figure map superimposed with image quality showing the microstructure and the local crystallographic orientation in the region of interest, b. Orientation density function in the region of interest relative to the loading direction and c. Crystallographic orientation relative to the loading direction of the nodules contained in the region of interest.

In order to consider the different behaviors of nodules and lamellar colonies, they were considered as different hexagonal close packed phases with different strength. Although the real behavior of colonies is more complex $[9,10]$, it enables to statistically account for the difference. Furthermore, as fatigue and dwell-fatigue are considered, crack initiation proceeds in alpha nodules. Since only the fields in these microstructural elements are discussed in the following, the introduced bias is limited. Prior studies reported a difference in strength between $5 \%$ and $25 \%$ with nodules [11,12]. Hence, it was arbitrarily set to $15 \%$ in the present work. Since the present study is based on the comparison of stress and strain fields resulting from different loadings, this should not affect the main conclusions of this work. The other parameters used for the crystal plasticity simulations are given in table 1.

Table 1. Material parameters used for crystal plasticity simulations $[5,13,14,15]$

\begin{tabular}{|c|c|c|c|c|c|c|c|c|c|c|}
\hline Parameter & $\mathrm{C}_{11}$ & $\mathrm{C}_{12}$ & $\mathrm{C}_{23}$ & $\mathrm{C}_{33}$ & $\mathrm{C}_{44}$ & $\begin{array}{l}\text { Basal } \\
\text { CRSS }\end{array}$ & $\begin{array}{l}\text { Prismatic } \\
\text { CRSS }\end{array}$ & $\begin{array}{c}\langle\mathbf{c}+\mathbf{a}\rangle \\
\text { pyramidal } \\
\text { CRSS }\end{array}$ & $\begin{array}{l}\text { Reference } \\
\text { shear rate }\end{array}$ & $\begin{array}{c}\text { Rate } \\
\text { sensitivity }\end{array}$ \\
\hline Value & $\begin{array}{c}162.4 \\
\mathrm{GPa}\end{array}$ & $92 \mathrm{GPa}$ & $69 \mathrm{GPa}$ & $\begin{array}{c}180.7 \\
\mathrm{GPa}\end{array}$ & $46.7 \mathrm{GPa}$ & $338 \mathrm{MPa}$ & $353 \mathrm{MPa}$ & $600 \mathrm{MPa}$ & $10^{-7} \mathrm{~s}^{-1}$ & 0.02 \\
\hline
\end{tabular}

Regarding the crystal plasticity model, the formulation described in [16] was implemented in an in-house code. The reader is referred to this article for further details. No self or latent hardening is considered here for simplification purposes. Furthermore, very low hardening rates were previously reported for Ti alloys [17]. No contribution of kinematic hardening was presently considered. Previous studies reported a good prediction of cyclic plasticity phenomena without such term [18].

The applied loading consists of $1 \mathrm{~s}$ loading to the maximum load and $1 \mathrm{~s}$ unloading to the minimum load. The minimum load is held for $1 \mathrm{~s}$. For fatigue testing, the maximum load is held for $1 \mathrm{~s}$ while it is held for $120 \mathrm{~s}$ for the dwell fatigue test. The maximum load was set to $90 \%$ of the yield stress, which was determined from a simulated tensile test at a strain rate of $10^{-2} \mathrm{~s}^{-1}$. The minimum load was set to $10 \%$ of the maximum load in order to obtain a load ratio (R) equal to 0.1 . A schematic representation of the applied loading waveform is available in [19].

\section{$\underline{\text { Results }}$}

The time step corresponding to the end of the first cycle and the time step corresponding to the end of the $62^{\text {nd }}$ cycle were considered for the dwell-fatigue and the fatigue tests respectively. Similar cumulated plastic strain magnitudes of $3.76 \times 10^{-4}$ were reached. 62 cycles of fatigue were thus needed to obtain the same cumulated plastic strain as a single cycle of dwell-fatigue. This highlights a significant contribution of creep at maximum load to the deformation in these conditions.

The YY component of the plastic strain tensor on the surface of the aggregate is presented in figure $3 \mathrm{a}$ and $\mathrm{b}$ for the dwell-fatigue and fatigue simulations respectively. High plastic strain magnitudes are mostly located in nodules. This feature is expected according to the high strength assigned to the transformed $\beta$ regions. Although similarities between both fields are obvious at first sight, subtle differences can be captured considering the local magnitudes. Both substantially higher and lower plastic strain magnitudes are noticed locally when comparing the fatigue plastic strain field to the dwell-fatigue field. Hence, a more heterogeneous plastic strain field is observed for the fatigue test. The difference between the fatigue and dwell fatigue plastic strain fields was calculated and is plotted in figure $3 \mathrm{c}$. Taking the dwell-fatigue test as a reference, higher magnitudes are noticed for the fatigue loading condition in nodules where the plastic strain is high. In contrast, lower magnitudes are noticed for the fatigue loading condition in nodules, or colonies, where the plastic strain is low.

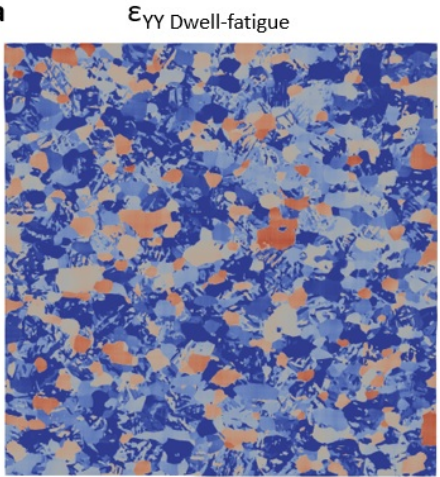

b

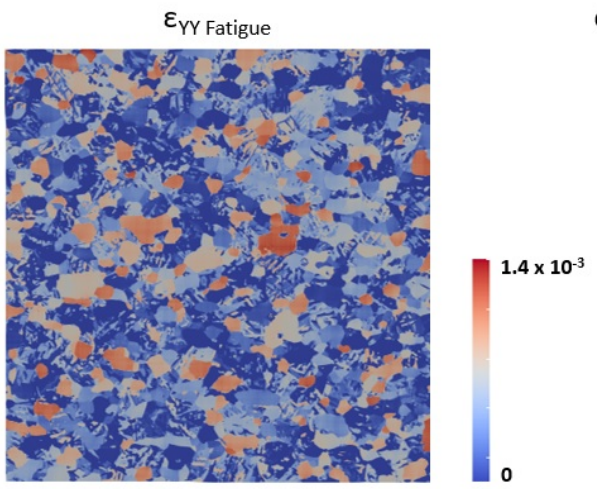

c $\Delta \varepsilon_{\mathrm{YY}}=\varepsilon_{\mathrm{YY} \text { Fatigue }}-\varepsilon_{\mathrm{YY} \text { Dwell-fatigue }}$

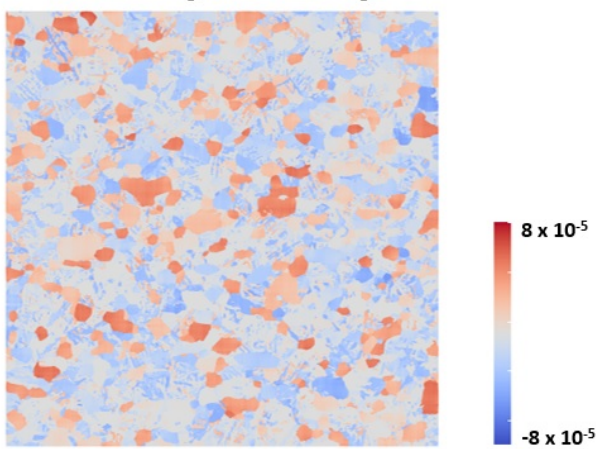

Figure 3 a. Magnitude of the YY component of the plastic strain tensor for a. the fatigue test, $b$. the dwell-fatigue test and $c$. the difference between the fields in figure 3 a. and $b$.

The magnitude of YY component of the stress tensor is shown in figure $4 \mathrm{a}$ and $\mathrm{b}$ for the dwell-fatigue and fatigue simulations respectively. Low stress magnitudes are mostly located in nodules. This feature is expected according to the higher strength assigned to the transformed $\beta$ regions and the plastic strain noticed in nodules that could favor stress relaxation. Although obvious similarities between both fields can be noticed at first sight, subtle difference can be captured considering the local magnitudes. A less heterogeneous stres field is observed for the fatigue test than for the dwell-fatigue test. In order to facilitate the comparison, the difference between the fatigue and dwell fatigue fields was calculated and plotted in figure 3c. Taking dwell-fatigue as the reference, microstructural elements can experience either higher or lower stress magnitudes under fatigue loading. The relation with microstructural features is discussed in the following paragraphs. 
a

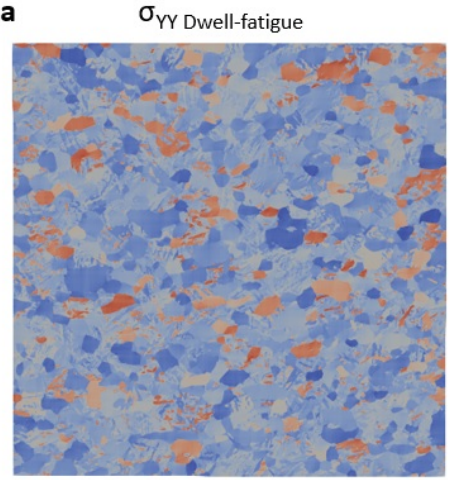

b

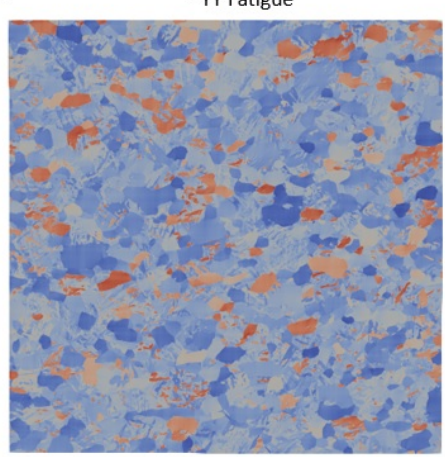

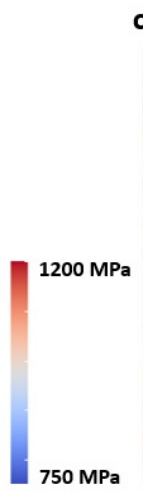

C $\Delta \sigma_{Y Y}=\sigma_{Y Y \text { Fatigue }}-\sigma_{Y Y \text { Dwell-fatigue }}$

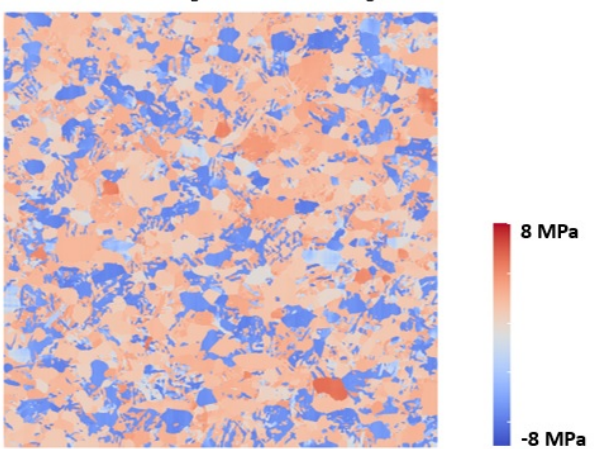

Figure 4 a. Magnitude of the YY component of the stress tensor for a. the fatigue test, b. the dwell-fatigue test and c. the difference between the fields in figure 3 a. and $b$.

The YY components of the plastic strain and stress tensors were averaged over each nodule and plotted in figure $5 \mathrm{a}$ and $\mathrm{c}$ with respect to the declination angle, which is defined as the angle between the c-axis of the hexagonal closed packed lattice and the loading axis. Considering the stress magnitudes first, high stress magnitudes are noticed for nodules with a declination angle lower than $20^{\circ}$. This is consistent with nodules where basal or prismatic slip could not operate owing to an insufficient resolved shear stress. Minimal values are reached at roughly $45^{\circ}$ and $90^{\circ}$. These values corresponds to Schmid factors of 0.5 for basal and prismatic slip. This suggests the occurrence of stress relaxation due to slip activity. This analysis is confirmed by the plot of figure $5 \mathrm{c}$. Indeed, high plastic strain magnitudes coincide with low stress magnitudes and high Schmid factors. The trends are very similar for fatigue and dwellfatigue loadings. However, a careful observation reveals a noticeable shift of some points. The difference between the stress magnitude calculated for fatigue and dwell-fatigue tests was computed. It unambiguously reveals that nodules, where no slip operates sustain a higher stress magnitude under dwell-fatigue loading while nodules where slip activity operates experience a higher stress magnitude under fatigue loading.
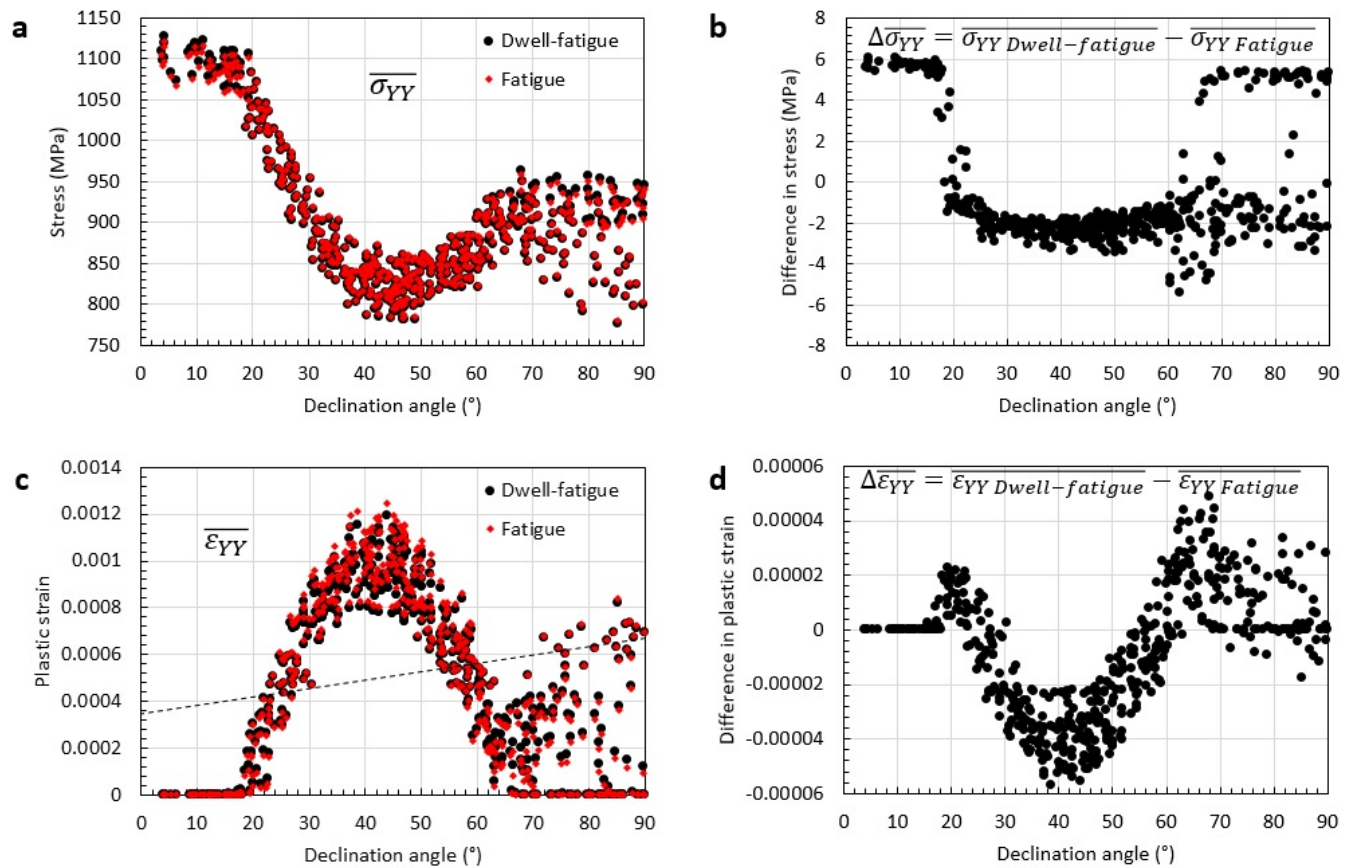

Figure 5 a. Magnitude of the YY component averaged in each nodule and plotted against the declination angle for a. the stress tensor and b. the plastic strain tensor. The difference between the values for the fatigue and the dwell-fatigue tests for each nodule regarding c. the stress and d. the plastic strain

The difference between the plastic strain magnitudes calculated for fatigue and dwell-fatigue tests was computed as well. It reveals that nodules where a low plastic strain is noticed sustain a higher plastic strain magnitude under dwell-fatigue loading while nodules where a high plastic strain magnitude is observed experience a higher plastic strain magnitude under fatigue loading. The frontier, where no significant difference between fatigue and dwell-fatigue is noticeable, is roughly indicated with a dashed line on figure $5 \mathrm{c}$.

\section{$\underline{\text { Discussion }}$}

The stress and plastic strain fields were compared at a similar cumulated plastic strain for fatigue and dwell-fatigue tests. Similar cumulated plastic strains were obtained after 1 cycle of dwell-fatigue versus 62 cycles of fatigue. Although a total load hold duration of $120 \mathrm{~s}$ was needed for the dwell-fatigue test, only $62 \mathrm{~s}$ were spent at maximum stress for fatigue loading. This reveals a clear effect of the cycling process on strain accumulation. In spite of very similar strain distribution observed, noticeable differences were evidenced. Regarding fatigue first, plastically deforming nodules experience both a higher stress magnitude and a higher plastic strain magnitude than under dwell-fatigue loadings for a similar macroscopic plastic strain magnitude. In contrast, under dwell fatigue loading, non-deforming nodules experience a higher stress magnitude and a reduced stress heterogeneity is observed.

If fatigue crack initiation can be described by a crack initiation criterion involving the plastic strain magnitude and the stress magnitude, such as the Fatemi-Socie fatigue indicator parameter [20], a lower macroscopic cumulated plastic strain magnitude is required for fatigue loadings than for dwell-fatigue loadings. Indeed, considering a similar macroscopic cumulated plastic strain value, both local stress and plastic strain magnitudes are higher in nodules potentially involved in fatigue crack initiation under fatigue loading than under dwell-fatigue loading. As a result a higher macroscopic plastic strain is necessary to reach the critical FIP value for crack initiation regarding dwell fatigue. This observation explains the high macroscopic plastic strain sustained under dwell fatigue loadings. Under fatigue loadings, a crack would have initiated for a similar macroscopic cumulated plastic strain. In addition, higher crack growth rates were reported in hard grains under dwell fatigue loadings [21]. At a similar cumulated plastic strain magnitude, hard grains experience a higher stress magnitude for the dwell-fatigue test, which acts as the crack opening stress. This is expected to facilitate the growth across these nodules. This is not happening in the specimen tested without the $120 \mathrm{~s}$ load hold.

\section{Conclusion}

The deformation behavior under fatigue and dwell-fatigue loadings of a realistic Ti-6Al-4V microstructural patch was simulated using a Fast-Fourier transforms-based crystal plasticity model. A special attention was paid to the stress and plastic strain fields, which are known to be critical for the prediction of fatigue crack initiation, at a similar plastic strain magnitude but different load hold durations. In spite of obvious similarities, noticeable differences were evidenced. Nodules well oriented for basal slip, which are potential fatigue crack initiation sites, experience higher stress and plastic strain magnitudes under fatigue loading than under dwell-fatigue loading. This point explains the occurrence of crack initiation at lower macroscopic plastic strain fatigue during fatigue tests than during dwell-fatigue tests. Furthermore, a higher stress magnitude was found in hard grains for dwell-fatigue testing. This observation, which is also verified at a similar number of cycles, suggests a contribution to the higher growth rates noticed for dwell-fatigue tests. 


\section{$\underline{\text { References }}$}

[1] J.A. Hall, Int. J. Fatigue 19 (1997) 23-37.

[2] W.J. Evans, M.R. Bache, Int. J. Fatigue 16 (1994) 443-452.

[3] A.L. Pilchak, J.C. Williams, Metall. Mater. Trans. A 42 (2011) 1000-1027.

[4] F.P.E. Dunne, D. Rugg, A. Walker, Int. J. Plasticity. 23 (2007) 1061-1083.

[5] M. Anahid, M.K. Samal, S. Ghosh, J. Mech. Phys. Solids 59 (2011) 2157-2176.

[6] F. Bridier, P. Villechaise, J. Mendez, Acta Mater. 56 (2008) 3951-3962.

[7] K. Le Biavant, S. Pommier, C. Prioul, Fat. Fract. Eng. Mater. Struct. 25 (2002) 527-545.

[8] J. Qiu, Y. Ma, J. Lei, Y. Liu, A. Huang, D. Rugg, R. Yang, Metall. Mater. Trans. A 45 (2014) 6075-6087.

[9] M.F. Savage, J. Tatalovich, M. Zupan, K.J. Hemker, M.J. Mills, Mater. Sci. Eng. A-Struct. 319-321 (2001) 398-403.

[10] G. Venkataramani, D. Deka, S. Ghosh, J. Eng. Mater. Tech. 128 (2006) 356.

[11] F. Bridier, D.L. McDowell, P. Villechaise, J. Mendez, Int. J. Plasticity 25 (2009) 1066-1082.

[12] D. Deka, D.S. Joseph, S. Ghosh, M.J. Mills, Metall. Mater. Trans. A 37 (2006) 1371-1388

[13] E.S. Fisher, C.J. Renken, Phys. Rev. 135 (1964) A482-A494. doi:10.1103/PhysRev.135.A482.

[14] S. Hémery, A. Nait-Ali, P. Villechaise, Mech. Mater. 109 (2017) 1-10.

[15] M. Kasemer, M.P. Echlin, J.C. Stinville, T.M. Pollock, P. Dawson, Acta Mater. 136 (2017) 288-302.

[16] R.A. Lebensohn, A.K. Kanjarla, P. Eisenlohr, Int. J. Plasticity. 32-33 (2012) 59-69.

[17] M.C. Brandes, M.J. Mills, J.C. Williams, Metall. Mater. Trans. A 41 (2010) 3463-3472.

[18] L.S. Tóth, A. Molinari, N. Zouhal, Mech. Mater. 32 (2000) 99-113.

[19] S. Hémery, P. Villechaise, Mater. Sci. Eng. A-Struct. 697 (2017) 177-183.

[20] A. Fatemi, D.F. Socie, Fat. Fract. Eng. Mater. Struct. 11 (1988) 149-165.

[21] A.L. Pilchak, Scripta Mater. 68 (2013) 277-280. 